\title{
Dentro de los portales bibliotecarios: flujo de trabajo en la automatización de bibliotecas
}

\author{
Tomás Saorín Pérez \\ Facultad de Ciencias de la Documentación \\ Universidad de Murcia \\ Jesús González Lorca \\ Fundación Séneca (Murcia) (1)
}

Es fácil rendirse, decir que lo has hecho lo mejor que has podido, y que otro trabaje en tu lugar. Lo importante es la determinación, la ambición. Dime cuál es tu motivación y entonces te diré quién eres. (Michael Jordan)

\subsection{Resumen}

Se dibujan las líneas principales para la elaboración del proyecto informativo público en línea de la biblioteca. En el contexto de la sociedad de la información, la biblioteca debe enfocarse conceptualmente como una Biblioteca Híbrida, donde se produce una constante evolución tecnológica y una convivencia entre información en múltiples sistemas de acceso, en la que lo digital tiende a adoptar un papel de integración y sustitución. La actual necesidad de disponer de un modelo de referencia para la evolución tecnológica del proyecto informativo de la biblioteca, partiendo desde un modelo de biblioteca automatizada orientado en exceso hacia los procesos técnicos internos, y la actual situación de complejidad y dispersión de los elementos tecnológicos implicados en la biblioteca (sistemas de gestión bibliotecaria, redes locales, OPACs, ofimática, bases de datos relacionales, webs, aplicaciones específicas de proceso, etc.) pasa por una revisión del concepto de automatización de bibliotecas, el cual, según nuestra propuesta, debe considerarse desde el punto de vista interno (procesos técnicos) como una herramienta de groupware y flujo de trabajo, y desde el punto de vista externo (información al usuario) como un portal Internet y una comunidad virtual. Se delimita el contenido y alcance de cada uno de los portales de la biblioteca, las pautas y estrategias para su desarrollo, así como el marco tecnológico global del sistema de información de la biblioteca.

Palabras clave: Automatización de bibliotecas. Automatización de procesos. Portales bibliotecarios. Flujos de trabajo. Sistemas Integrados de Gestión Bibliotecaria. 


\subsection{Abstract}

The main lines for the design of a library online public information project are drawn. In the context of the information society, the library must be understood as a hybrid library, characterized by a constant technological evolution and the coexistence of multiple systems of access to information in multiple systems of access, and in which digital information adopts mainly a role of integration and substitution. Current library automation must evolve from a model basically oriented to the internal technical processes towards a newer and more integrated concept, and must address the present situation of complexity and dispersion of the different library technologies, i. g., library systems, local networks, OPACs, office automation, relational databases, webs, specific applications, etc. Library automation must be considered, from an internal point of view (technical processes), as a groupware and workflow project, and, from an external point of view (information for the users), as an Internet portal and a virtual community project. The content and scope of the different levels of library portals, and the guidelines, strategies and the global technological frame for development of a library information system are delimited.

Keywords: Library automation. Process automation. Library portals. Workflow. Library systems.

\section{Los portales bibliotecarios}

\subsection{Portales y comunidades virtuales}

La presencia activa de las bibliotecas en la web, mediante proyectos cada vez más amplios, donde se potencia su actividad informativa y de servicio, hace cada vez más común hablar de portales, destacando el nivel de madurez que comienzan a demostrar ciertos servicios en línea individuales y cooperativos. El resultado más visible de la evolución de la automatización de bibliotecas hacia sistemas que soportan la extensión de los servicios de las bibliotecas mediante el uso de todo tipo de redes es lo que denominaremos genéricamente "portal".

Un portal se entiende como una agregación de contenidos y funcionalidades, organizado para facilitar el acceso a la información, con vocación de servir al usuario como punto personalizado de entrada a la red Internet. Ese punto de entrada se caracteriza por la concentración de información, la extensa gama de servicios y productos, y la sus amplitud de sus objetivos. Los portales de información satisfacen en parte una necesidad de orientación y concentración de información, pero las comunidades virtuales permiten acercar la visión de los usuarios a los servicios documentales ofrecidos. Los portales han tenido un impacto importante en la evolución de los grandes servicios de búsqueda en Internet y en los servicios de información especializados en su afán por captar usuarios respondiendo a todo tipo

Scire. $9: 2$ (jul.-dic. 2003) 37-59. 
de necesidades de información y servicio. Por lo general se distingue entre grandes portales horizontales - de ámbito general y gran audiencia - y portales especializados - corporativos para organizaciones concretas, y verticales para sectores empresariales (García Gómez, 2001; Barò, Ontalba y Ruipérez, 2001).

Enfocar la web de la biblioteca como una comunidad virtual supone tratar de poner al usuario y sus necesidades de información en el centro del proyecto. Implica un uso del portal electrónico por los usuarios tradicionales de la biblioteca (Saorín Pérez y Sánchez Arce, 2001). Ambos enfoques son complementarios, de manera que (Saorín Pérez; García Gómez, 2001):

Los portales y las comunidades virtuales existirán mientras tengan sentido para un grupo de personas. Si el crecimiento de Internet se debía a la necesidad de compartir información, en la actualidad los usuarios buscamos espacios para compartir necesidades de información. Las tecnologías y recursos construidos para ello se denominan ahora portales. Los usuarios que interactúan en ellos, comunidades virtuales.

Podemos decir que la biblioteca crea una comunidad virtual de usuarios que comparten necesidades de información, por medio de un portal que concentra servicios de información y comunicación. Para considerar a la web de una biblioteca como un portal, tendrá que alcanzar un cierto nivel de exigencia, sobre el que podemos apuntar, como esquema de referencia, la propuesta para el análisis de comunidades virtuales especializadas de Fernández Sánchez, Fernández Morales y Maldonado Martínez (2000), que contempla cuatro elementos:

- Aspectos técnicos de acceso a la información: acceso temático, mapas del web, buscadores internos, buscadores externos, elementos multimedia.

- Documentación referencial: bases de datos bibliográficas propias y externas, catálogos de libros, directorios de expertos, de organismos, web analizadas, convocatorias, etc.

- Documentación de contenido: revistas propias y externas, textos electrónicos, normativa, páginas temáticas, etc.

- Servicios: perfiles para la alerta técnica, asesoría legal, foros de discusión, correo electrónico, alojamiento páginas personales, etc.

\subsection{Niveles de los portales en la biblioteca híbrida}

$\mathrm{Al}$ entender la biblioteca actual como una biblioteca híbrida, donde conviven en un proceso dialéctico y complejo de integración, entornos tradicionales y digitales, tanto en las colecciones, los servicios, las herramientas de información, los espacios y los usuarios, debemos considerar con atención los distintos contexto de servicio (Crawford y Gorman, 1995; Oppenheim y Smithson, 1999; Rusbridge, 1998; Moreiro González, 1999). La biblioteca híbrida es un espacio real en que usuarios presenciales acceden a información y servicios de manera

Scire. $9: 2$ (jul.-dic. 2003) 37-59. 
tradicional (mostradores, peticiones) o de forma electrónica (OPACs, consulta CD-ROM en red, acceso Internet), al mismo tiempo que un espacio virtual articulado en torno a sus diferentes portales abiertos en diversos contextos de uso: fuera de la biblioteca (web), desde otras bibliotecas (extranet) y desde dentro de la propia biblioteca (intranet) (Saorín, 2001).

Mientras que la tecnología está en el centro de todas las reflexiones, las personas continúan viviendo en lugares reales y usando objetos reales (Brophy, 2001). La biblioteca híbrida debe ser gestionada también como un espacio físico en el que tiene lugar una parte importante del servicio, y dotado de un sentido relevante en la vida de la comunidad. La biblioteca es un lugar físico y un lugar en la red. La biblioteca puede ser usada a distancia desde ordenadores conectados a la red, al mismo tiempo que desde las salas de la biblioteca podemos acceder a distancia a recursos de información en Internet o documentos electrónicos en la red. La biblioteca puede actuar en la red mediante su propio portal, o bien integrada en proyectos cooperativos, y su web puede ser pasarela para el acceso a esos recursos y servicios desarrollados en cooperación. En respuesta a estas situaciones, presentamos los portales en cinco niveles: a) el portal público en Internet - la web de la biblioteca - ; b) el portal público en intranet - la biblioteca electrónica-; c) el portal público en CD - la biblioteca portátil-; d) el portal extranet - la Biblioteca-Red - ; y e) el portal interno para el personal - entorno de trabajo y servicio. Estos cinco niveles se examinan con detalle a continuación.

\subsubsection{Portal público en Internet: la web de la biblioteca}

El portal en Internet es la piedra angular de la política de servicios electrónicos de la biblioteca, puesto que abre la biblioteca al exterior, haciendo accesible su contenido informativo a una comunidad de usuarios abierta. En los inicios las bibliotecas estuvieron presentes en Internet mediante el acceso aislado a sus catálogos, y actualmente casi cualquier biblioteca de cierta importancia posee su propia web.

El proyecto web forma parte de la estrategia de información y servicios de la biblioteca, y no es tan solo una mera presencia institucional en la red. Posee un valor para la organización y no es una actividad secundaria de la biblioteca. Dado el panorama enormemente competitivo que existe en Internet, donde proliferan servicios de información altamente imaginativos y con gran potencia de atracción de usuarios y recuperación de información, las bibliotecas se encuentran con el reto de demostrar la vigencia de su papel en la sociedad digital. Para que el proyecto web pueda ser un éxito, en las circunstancias actuales de Internet, deberá:

- Ser un foco multiplicador de la información de la biblioteca y un banco de pruebas de proyectos de ampliación de sus contenidos, de manera que genere recursos de interés únicos para el usuario, tales como revistas y dossieres de temas locales, información única...

Scire. $9: 2$ (jul.-dic. 2003) 37-59. 
- Tener diseño e imagen profesional, especialmente en lo relativo a la entrada al web, la consistencia de las plantillas de documentos y a los contenidos multimedia de carácter didáctico y de formación de usuarios.

- Poseer una dirección Internet de calidad, mnemotécnica y directa.

- Proporcionar a los usuarios una "red electrónica de información", entendida como direcciones de correo electrónico estables para los servicios básicos.

- Dar valor añadido frente a los buscadores genéricos, proporcionando acceso a la información almacenada en documentos impresos y al asesoramiento humano en la búsqueda. El valor añadido se puede entender también como un aumento de la información digital ofrecida - extractos, reseñas, imágenes, documentos completos - , así como en relación al desarrollo de bases de datos específicas para necesidades concretas carentes de recursos apropiados en Internet, ayudando a rellenar lagunas.

- Tener en cuenta en su diseño técnico las condiciones de accesibilidad y utilización, así como los procedimientos dinámicos de gestión, basados en una arquitectura de datos potente residente en la propia aplicación de gestión bibliotecaria.

- Potenciarse mediante la colaboración en Internet.

\subsubsection{Portal público en intranet: la biblioteca electrónica}

Este portal es fundamental para hacer las instalaciones de la biblioteca un espacio informativo comunitario mejorado. En primera instancia dependerá de la estrategia seguida en la configuración de la red multimedia para usuarios. En segunda instancia dependerá de cómo se ofrece la información al usuario de esos servicios (2).

El portal público en intranet será la puerta de entrada a los ordenadores de servicio al usuario en las salas de la biblioteca; y, por lo tanto, tendrá un papel muy importante en el uso que se haga de la red Internet. El portal intranet permite a los usuarios el acceso interactivo a través de ordenadores a recursos y servicios de información avanzados, dentro de las instalaciones de la biblioteca. Aplica las posibilidades de la tecnología en el acceso a la información a los usuarios locales, potenciando la organización física de la colección en las salas. Permite buscar en la colección impresa usando técnicas digitales, así como acceder a información de resumen, guías, etc. Actúa al mismo tiempo como puerta de entrada a la información electrónica de la biblioteca y a la información pública en Internet. Es el entorno donde se materializa el papel de la biblioteca como orientadora. $\mathrm{Al}$ mismo tiempo estimula el uso de la web de la biblioteca dentro de la biblioteca, no solo fuera. Es el medio de explotación de la colección electrónica. Está vinculada con el desarrollo de la colección, pues la intranet es el medio óptimo para la oferta de la colección multimedia propia de la biblioteca (CD-ROM y DVD)

Scire. $9: 2$ (jul.-dic. 2003) 37-59. 
(3) y el acceso a las suscripciones en línea que se poseen. También es la vía natural de acceso a la biblioteca aumentada en extranet. A grandes rasgos puede ser muy similar al web de la biblioteca, pero con mayores posibilidades y con vistas especializadas adaptadas a la ubicación de cada punto informático en la biblioteca - por ejemplo, entradas y perfiles diferentes para ordenadores situados en la sección infantil o en la mediateca.

\subsubsection{El portal público en CD: la biblioteca portátil}

En la era de Internet a veces se olvida la enorme importancia que sigue teniendo el documento como objeto material tangible, cercano al entorno inmediato del usuario, y con posibilidades de portabilidad. Por lo tanto se plantea como requerimiento a la estrategia de información electrónica de la biblioteca la creación de una versión del portal público Internet almacenable en los dispositivos ópticos más comunes (CD-ROM y DVD-ROM). Responde a una necesidad de apropiación de la información. Ofrece como ventajas añadidas, además de las comentadas de portabilidad y carácter tangible, la proyección de una imagen positiva de la biblioteca a través del soporte de la publicación. Es más costoso de producir, pues conlleva costes de duplicado y actualización, pero puede entenderse como una herramienta informativa práctica por sí misma, al mismo tiempo que un medio de promoción de la biblioteca. Actualmente se tiende a un modelo reduccionista del uso del $\mathrm{CD}$, solo para la edición de catálogos de bibliotecas, produciéndose algo similar a cuando se reduce el sistema de información público de la biblioteca exclusivamente al OPAC. Por el contrario, este CD contendrá el máximo de información corporativa y documental, y será el entorno óptimo para la incorporación de contenidos multimedia con altos requerimientos. Sus contenidos incluirán la información institucional básica — guía de la biblioteca-; la memoria de actividades, la memoria institucional y el proyecto de la biblioteca; catálogos bibliográficos y otras bases de datos documentales; recopilación de materiales informativos realizados - guías de recursos, hojas informativas, folletos, etc.; información visual - fotos, vídeos, planos, etc.; tutoriales multimedia; y el enlace con la web de la biblioteca.

\subsubsection{Portal extranet: la Biblioteca-Red}

Esta acepción de portal es la comúnmente aplicada en Internet. Se entiende por portal algo más que web institucional, creando proyectos de información que ponen en primer plano el área temática de interés, aglutinando información producida y gestionada por diversos centros y departamentos, con el fin de simplificar su acceso y aumentar la captación de usuarios. En muchos casos supone la creación de nuevas estructuras organizativas para la gestión de la documentación - que ayuda a un proceso gradual de transición de los centros de documentación tradicionales al nuevo contexto - , mientras que en otras puede actuar simplemente como pasarela

Scire. $9: 2$ (jul.-dic. 2003) 37-59. 
a recursos documentales finales. Este carácter de oferta de servicios y productos de información a través de la red, independientemente de las instituciones reales que la gestionan y producen, es a veces invocado al usar el término biblioteca virtual, entendida como biblioteca distribuida. Por portal extranet entendemos no un portal individual, sino el conjunto de prestaciones de los portales públicos de la biblioteca (en Internet o Intranet) que se basan en el funcionamiento en cooperación con otras bibliotecas o entidades con las que forma una red.

La extranet amplia la capacidad de la red de la biblioteca, respondiendo al reto de la "biblioteca sin paredes", puesto que desde el portal de la biblioteca se accede a recursos más amplios que los posee por sí misma la biblioteca como unidad. En este sentido se habla a veces de biblioteca virtual, puesto que no existe más que ampliación de recursos de una biblioteca concreta. En este trabajos preferimos hablar de biblioteca-red; biblioteca que forma parte de muchas redes de información, a las que da acceso, siendo difícil distinguir donde termina exactamente el alcance de esta biblioteca aumentada.

La conceptualización de la biblioteca como un centro comunitario limita las posibilidades de construcción de bibliotecas virtuales, basadas en el funcionamiento conjunto de varias bibliotecas en red. El usuario busca la información a través de su biblioteca local, y es ésta la que le da acceso a recursos ampliados externos la biblioteca en sí. La extranet es la vía para un crecimiento de las bibliotecas basado en la cooperación bibliotecaria, concretada en servicios y productos informativos que se apoyan en las tecnologías de la información y comunicación. Tiene una doble dimensión: el acceso a servicios virtuales cooperativos y la extensión de la red interna de la biblioteca. Permite la creación de una intranet virtual entre bibliotecas para compartir el acceso a la información y los documentos, de manera que el usuario de una biblioteca sea al mismo tiempo usuario de toda la información contenida en las intranets de las bibliotecas que forman la red. La orientación extranet puede realizarse no solo entre bibliotecas, sino también con otros agentes, como pueden ser librerías, distribuidores de información, buscadores, etc. Además puede aplicarse también a los procesos técnicos, de modo que los proyectos informativos y el tratamiento documental se realizan también en cooperación. Obliga al diseño de sistemas de información distribuidos, que puedan ser usados de manera compartida por todos los usurarios de los servicios de la biblioteca. Estos acceden al recurso o servicio de información desde el portal de su biblioteca, siendo para ellos transparente la forma en la que se gestiona internamente la información y el modo en que participa cada biblioteca en el sistema.

\subsubsection{Portal interno para el personal: entorno de trabajo y servicio}

El personal que presta sus servicios y gestiona la biblioteca también posee una visión exclusiva de la biblioteca y sus recursos. La biblioteca para su gestión efecti-

Scire. $9: 2$ (jul.-dic. 2003) 37-59. 
va como organización productora de servicios requiere de herramientas avanzadas para la gestión del conocimiento y el apoyo al trabajo intelectual y los procesos técnicos asociados. Este portal sería, precisamente, la entrada a la biblioteca por parte del personal técnico y de servicio al usuario, adaptado a sus perfiles concretos de actividad, y con funcionalidades extendidas de acceso a la información. Al igual que el portal intranet para usuarios, se adapta al contexto de cada servicio (infantil, prensa, legislación, mediateca, etc.), pero desde el punto de vista de la atención del servicio. Ambos formarían las dos caras de una misma pieza. Este portal surge de la consideración de la biblioteca como una organización compleja, cuya gestión debe ser abordada con las modernas técnicas de dirección empresarial, y cuyos trabajos técnicos se apoyan en herramientas para potenciar la productividad, la adaptación del usuario, y el aprovechamiento del conocimiento. La concepción de este portal está estrechamente vinculada a las prestaciones de la red local de servicios ofimáticos, donde se trabaja con documentos e informes, compartiendo depósitos documentales mediante la comunicación electrónica.

El portal interno de la biblioteca se articula en tres subproyectos: a) dirección y diseño de procesos organizacionales; b) planificación y normalización de procesos técnicos; y c) atención al usuario. Además pueden aplicarse los contenidos del enfoque extranet para potenciar estos portales internos, siendo recomendable que funcionen de modo compartido entre redes de bibliotecas, ayudando a un espacio bibliotecario mejor vertebrado.

\section{La doble automatización de la biblioteca: servicios al público y procesos técnicos}

El objetivo final de los SIGB es la producción de entornos bibliotecarios automatizados de altas prestaciones en servicios e información para el usuario, mediante la generación de bases de datos documentales, la organización de la información y su difusión. El portal, la parte pública de la biblioteca automatizada, estará en el centro de los procesos técnicos, orientando su realización en función del resultado para el usuario. Podemos decir que el OPAC corresponde a una primera generación de sistemas de información públicos de la biblioteca, cuya función se amplia y revisa en los portales bibliotecarios, los cuales constituyen la segunda generación.

\subsection{La biblioteca como organización y servicio}

La automatización debe aportar valor añadido a cada una de las funciones de la biblioteca. Basándonos en una definición amplia de biblioteca - ser un espacio de trabajo, estudio y convivencia; dar asesoramiento y consultoría sobre información; ser foco de cultura y ocio creativo; contribuir a la formación de los usuarios, ser un servicio de información; proporcionar documentos - , podemos

Scire. $9: 2$ (jul.-dic. 2003) 37-59. 
reflexionar sobre en qué medida el actual modelo de automatización de bibliotecas produce una visión sesgada de la organización biblioteca.

La biblioteca se puede entender tanto desde su funcionalidad externa - servicio de información - como desde su estructuración interna como organización que los produce. Desde el punto de vista del servicio básico de la biblioteca como centro de información, hay cuatro elementos sobre los que tenemos que focalizar nuestra atención: sistema deinformación, colección, servicios y usuarios. Desde un punto de vista de la biblioteca como organización que produce unos servicios, hay otros tres elementos a contemplar con prioridad: sistemas de información, procedimientos y personal. El portal es el nexo entre ambas dimensiones (fig. 1). Une el mundo real con el virtual. Así, el usuario percibe la biblioteca a través del servicio digital, accede a ella remotamente - de forma virtual - tanto como físicamente - real-: por ejemplo, busca en el catálogo informatizado, pero saca en préstamo el libro físico.

\subsection{Automatización de la biblioteca en el contexto digital}

La automatización de bibliotecas se puede concebir como una de las muchas formas que adopta la gestión documental en las organizaciones. Un modelo adaptado a las necesidades modelo de una biblioteca. Sin embargo, como hemos visto, la creciente complejidad y diversidad de los servicios y recursos documentales de las instituciones, hace que la gestión documental en bibliotecas demande una apertura hacia otros escenarios.

Trataremos aquellos dos grandes campos que permiten enclavar a los SIGB dentro del espectro de aplicaciones de gestión documental: los entornos ofimáticos de trabajo y el workflow. En estos dos contextos el término integración adquiere una nueva dimensión, que, aunque ha sido ampliamente usado en el

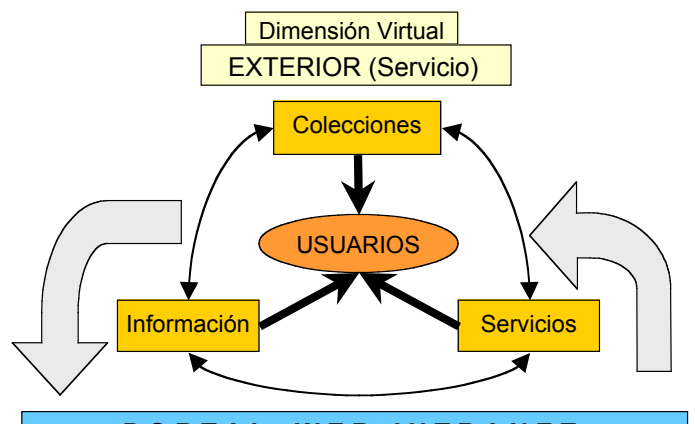

PORTAL WEB-INTRANET

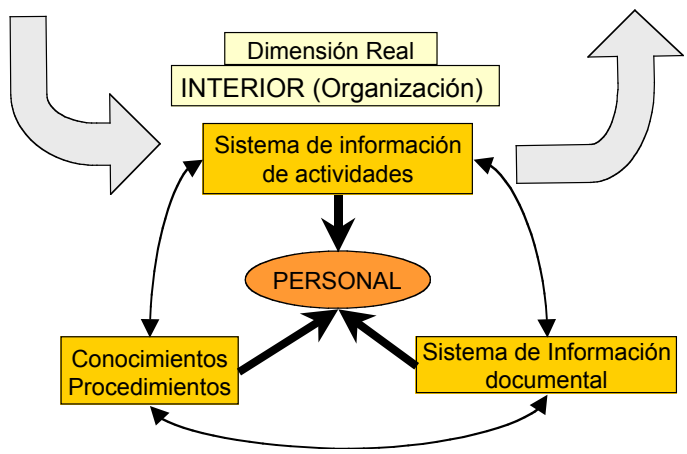

Fig. 1. Las dos dimensiones en torno al

Scire. $9: 2$ (jul.-dic. 2003) 37-59. portal de la biblioteca 
contexto bibliotecario desde hace más de veinticinco años, su verdadero alcance varía según los casos y aspiraciones. Independientemente del nivel de integración que podamos identificar en un sistema, es indudable que existe una necesidad conceptual de compenetración entre todos los agentes y sistemas implicados en el proceso informativo de las bibliotecas. Integración ha sido un término clave en el campo de la automatización de bibliotecas, especialmente significando multifuncionalidad y estructura de datos unificada. Pero integración también tiene un significado más amplio, que denota un auténtico reto en el contexto extendido de la biblioteca digital e Internet, que implica que todas las operaciones informatizadas de la biblioteca han sido planificadas cuidadosamente, y que todos los módulos o subsistemas encajan armoniosamente, de manera que las operaciones de base (la informática de sistemas) no constituyan una preocupación para los gestores, los cuales se ocupan básicamente de explotar y gestionar la información. Se define la situación actual como "integración de sistemas" más que "sistemas integrados", puesto que un número de creciente de áreas de actividad bibliotecaria funcionan ajenos a la aplicación de gestión bibliotecaria tradicional (Jacqueson, 1995). Las premisas y modelos del nuevo concepto son las siguientes:

- La automatización de bibliotecas debe englobar todos los procesos en los que están implicada la tecnología y en los que ésta puede aportar valor añadido a cada uno de los servicios de la biblioteca.

- Los SIGB clásicos comparten una estructura de módulos similar. adquisiciones, catalogación, opac, publicaciones seriadas, etc.

- La automatización se centra en el servicio, no en los procesos técnicos.

- La complejidad de la aplicación de la tecnología en las bibliotecas ha desbordado las posibilidades de los SIGB, produciéndose una situación de Integración de sistemas más que de sistemas integrados.

- Los principales programas a nivel internacional ha sido sometidos a un profundo rediseño para su adaptación a las nuevas demandas del entorno tecnológico actual, que rebasa el modelo conceptual subyacente a los clásicos SIGB, con funciones tales como la gestión de bibliotecas digitales, la digitalización o las pasarelas Internet a servicios comerciales.

- En el mercado de productos para la automatización se observa una tendencia a la convergencia entre empresas de software y empresas de contenidos.

- La incorporación de la tecnología forma parte de la gestión estratégica de la biblioteca, puesto que incide de lleno en las posibilidades del servicio y en su calidad, al mismo tiempo que en la mejora de procesos.

- La estrategia de los grandes grupos de distribución bibliográfica pasa por la Internet, la integración de fuentes de información, la colaboración estrecha

Scire. $9: 2$ (jul.-dic. 2003) 37-59. 
con los productores de contenidos y la universalización del acceso a la información.

- Las prestaciones básicas de las aplicaciones de gestión bibliotecaria inciden fuertemente sobre el modelo de servicio ofertado por las bibliotecas.

- La construcción de bibliotecas digitales - servicios de biblioteca con una oferta amplia de recursos informativos-documentales y servicios al usuario accesibles en red- deberá estar basada en herramientas de automatización integrales basadas en una modelización global de la biblioteca.

Antes de proceder a un análisis funcional más detenido, es necesario precisar que nos encontramos ante un SIA que combina estructuras de datos e información documental, por lo que debe ser conceptualizado, tal y como propone Moya, siguiendo los trabajos de Kemp, a partir de un modelo híbrido, que combina el acercamiento tradicional de las técnicas de recuperación de información con la gestión de bases de datos relacionales (Moya, 1995). La mayor de los programas clásicos de automatización de bibliotecas siguen la misma línea en cuanto a su estructura funcional básica. Para definir un SIGB es necesario realizar atender cuatro dimensiones relativas a un análisis orgánico y funcional (Moya, 1995): estructura organizativa de la biblioteca, actividades, procesos sobre información estructurada, y procesos sobre información no estructurada. Un SIGB extendido se estructura en los siguientes módulos operativos:

1. Gestión conocimiento corporativo (GCC): entorno de apoyo al trabajo técnico bibliotecario.

2. Gestión documental de la colección (GDC): operaciones de tratamiento documental, diseño de bases de datos, almacenamiento de documentos digitales y codificación de información.

3. Diseño de servicios y productos informativos (DSPI): definición de los parámetros de servicios al usuario y procedimientos asociados, y de los productos informativos de consulta y difusión de información.

4. Gestión de portales de servicio (GPS): diseño de espacios de atención al usuario, en acceso directo o mediado, en entornos presenciales y digitales, bien como puntos de servicio interactivos, bien como apoyo a los servicios de atención personal.

Todas las actividades relacionadas con la automatización de bibliotecas se han visto potenciadas y al mismo tiempo replanteadas con la extensión de Internet (Jacquesson, 1995). El primer signo visible ha sido la puesta en marcha de los OPAC accesibles en Internet, en un principio mediante conexión telnet y posteriormente con pasarelas web. La posibilidad de interconexión entre redes locales y la apertura al público de los sistemas de información hace de Internet un con-

Scire. $9: 2$ (jul.-dic. 2003) 37-59. 


\section{Objetivos del SIGB extendido}

\begin{tabular}{|c|c|}
\hline $\begin{array}{l}\text { 1. Automatizar } \\
\text { la producción } \\
\text { del sistema de } \\
\text { información público } \\
\text { de la biblioteca o } \\
\text { portal }\end{array}$ & $\begin{array}{l}\text { 01) Diseño y gestión del web. } \\
\text { 02) Diseño de las utilidades de navegación y ayuda. } \\
\text { 03) Edición electrónica dinámica. } \\
\text { 04) Definición del interfaz de recuperación y acceso a la } \\
\text { información. } \\
\text { 05) Integración del OPAC. } \\
\text { 06) Diseño de agentes de apoyo al usuario. }\end{array}$ \\
\hline $\begin{array}{l}\text { 2. Automatizar la } \\
\text { producción de las } \\
\text { bases de datos } \\
\text { documentales de la } \\
\text { biblioteca }\end{array}$ & $\begin{array}{l}\text { 07) Definición de bases de datos y productos documentales } \\
\text { propios. } \\
\text { 08) Integración de sistemas documentales externos. } \\
\text { 09) Gestión integrada de puntos de acceso para la navegación } \\
\text { (Autoridades). } \\
\text { 10) Integración de documentos en línea o digitalizados. }\end{array}$ \\
\hline $\begin{array}{l}\text { 3. Automatizar la } \\
\text { gestión de servicios } \\
\text { al usuario de la } \\
\text { biblioteca. }\end{array}$ & $\begin{array}{l}\text { 11) Servicios básicos de la colección: préstamo, consulta, } \\
\text { accesos. } \\
\text { 12) Servicios espaciales de la biblioteca: uso de salas, } \\
\text { recursos y equipamientos. } \\
\text { 13) Definición de parámetros dinámicos de usuarios. }\end{array}$ \\
\hline $\begin{array}{l}\text { 4. Gestionar el flujo } \\
\text { de trabajo interno de } \\
\text { la biblioteca }\end{array}$ & $\begin{array}{l}\text { 14) Control del proceso técnico, selección y adquisición. } \\
\text { 15) Información estadística del sistema. }\end{array}$ \\
\hline $\begin{array}{l}\text { 5. Automatizar el } \\
\text { sistema de gestión } \\
\text { del conocimiento } \\
\text { interno de la } \\
\text { biblioteca }\end{array}$ & $\begin{array}{l}\text { 16) Creación de bases de datos de procedimientos y } \\
\text { manuales. } \\
\text { 17) Gestión de herramientas internas de comunicación } \\
\text { organizativa. } \\
\text { 18) Difusión selectiva de información profesional. } \\
\text { 19) Apoyo al desarrollo de proyectos y trabajo en equipo. }\end{array}$ \\
\hline
\end{tabular}

Tabla I. Objetivos del SIGB extendido

texto de posibilidades inmensas. Al mismo tiempo, aparece una marea inagotable de nuevas fuentes de información en la misma red, que ocupan un lugar social destacable, rompiendo las tradicionales fronteras entre tipologías documentales. Internet supone además que en la misma red de información se encuentran los documentos y los sistemas documentales que dan acceso a ellos. Por lo tanto, se genera un espacio nuevo, en el que la red ofrece la posibilidad de construir bibliotecas virtuales basadas en colecciones y sistemas de información digitales.

¿Con qué modelos tecnológicos, con qué tipología de aplicaciones, podemos enfocar estos retos planteados para los sistemas de automatización integral de Bibliotecas? Esta situación no es exclusiva de las bibliotecas, sino que la mayor parte de las organizaciones actuales, que habían automatizado sus procesos más 


\begin{tabular}{|l|l|}
\hline \multicolumn{2}{|l|}{ Principales transformaciones en marcha } \\
\hline Sistema de información general & Sistema de información personalizado \\
\hline SIGB individual & SIGB colectivo \\
\hline SID referencial & SID texto completo \\
\hline SID cerrado & SID abierto. Servicios de distribuidores y documentos \\
\hline SID bibliográfico & SID multipropósito \\
\hline Documentos papel & Colección digital distribuida \\
\hline OPAC sala & Puntos de servicio Interactivo \\
\hline Sala de consulta & Sala de consulta digital \\
\hline Biblioteca electrónica & Biblioteca digital / virtual \\
\hline SID Público-OPAC & SID Público-WWW \\
\hline Recuperación de información & Knowledge discovering \\
\hline Sistema consulta & Sistema de difusión. Comunidad virtual \\
\hline Biblioteca unidad & $\begin{array}{l}\text { Biblioteca red. Participación en productos y servi- } \\
\text { cios cooperativos en línea }\end{array}$ \\
\hline Gestión del préstamo & Gestión de servicios de la biblioteca \\
\hline
\end{tabular}

Tabla II. Transformaciones más relevantes en curso

estructurados, han hecho un uso de la informática más amplio, como herramienta de gestión del conocimiento, comunicación, elaboración de documentos y trabajo en grupo. Puesto que nos movemos en un entorno de oficina o administrativo, conviene conocer cómo se están aplicando las tecnologías para automatizar sus procesos y esquemas de funcionamiento, compartir información, almacenamiento de conocimiento corporativo. Este ámbito de la aplicación de las tecnologías al trabajo de oficina se denomina groupware, y pensamos que es un marco de referencia desde el que debe revisarse la definición de los sistemas de automatización de bibliotecas. El groupware gira en torno a tres ejes de acción, que se encuentran presentes en mayor o menor medida, en toda organización de servicios: comunicación, colaboración y Coordincción. La tecnología de automatización de flujo de trabajo se ocupa principalmente de este último aspecto, integrando la comunicación y la colaboración en un mismo entorno para la realización de trabajos estructurados, mientras que el groupware contempla también aquellos procesos no estructurados (González Lorca, 2001).

Scire. $9: 2$ (jul.-dic. 2003) 37-59. 
En resumen, el sistema-biblioteca se caracteriza por su complejidad, dados sus componentes, configuración, interacción, objetivos, etc. Aquí es donde el concepto de "integración" adquiere gran relevancia. Según la integración alcanzada en el entorno biblioteca, así será la compenetración de sus componentes en aras del dinamismo y eficiencia de los procesos - servicios - a realizar. A continuación se analizan los aspectos específicamente referidos a los flujos de trabajo.

\section{La tecnología de flujo de trabajo como soporte de los procesos bibliotecarios}

\subsection{Definición de flujo de trabajo}

La Workflow Management Coalition (WfMC) (1998), organización internacional sin fines lucrativos, compuesta por distribuidores, analistas y usuarios de flujos de trabajo, cuyo objetivo es normalizar su terminología, conectividad e interoperatibilidad, define workflow como:

[...] la automatización de un proceso de negocio, total o parcialmente, en el que información de cualquier tipología llega al usuario adecuado en el momento adecuado, en base a un conjunto de reglas inteligentes, que permite que la mayoría del trabajo sea efectuado informáticamente, mientras que las personas se ocupan solamente de las excepciones.

Así, un sistema de workflow es considerado como "un sistema que automatiza procesos de negocio mediante la gestión de la secuencia de actividades que los forman, y la invocación de los recursos apropiados para cada una de ellas” (Hollingsworth, 1995).

\subsection{Las tres dimensiones de un flujo de trabajo: el cubo del workflow}

Para abordar el tema de la aplicación de los flujos de trabajo a entornos organizativos o empresariales, es prioritario conocer su filosofía de funcionamiento. A este respecto, destaca un informe realizado en la década de los 90 por IBM, en el que se presentan los tres ejes sobre los que gira la función de definición de un flujo de trabajo para automatizar un proceso:

a) El proceso (qué se desarrolla): Un proceso es "una visión formal de un proceso de negocio, representada como un conjunto coordinado de actividades (en serie y/o en paralelo) conectadas para alcanzar un objetivo común" (WfMC, 1999). Un ejemplo de proceso lo encontramos en la figura 2. Una actividad sería entonces, "la acción realizada por una persona o un recurso en un lugar determinado”. La actividad constituye la unidad mínima de trabajo. El núcleo del sistema de flujo de trabajo es, por tanto, el proceso al que se aplica, puesto que su fin radica en la automatización de la secuencia de actividades que lo componen.

b) La organización (quién desarrolla): Esta dimensión se refiere a la estructura de recursos humanos de la organización. A través de ella, es posible definir quién 


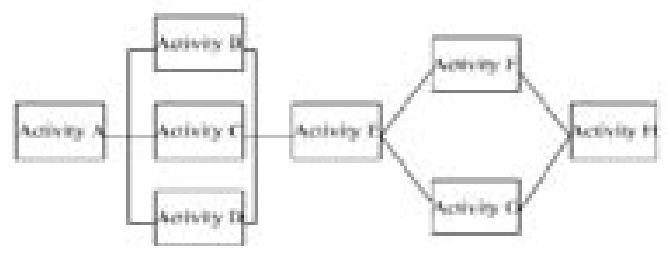

Fig. 2. Ejemplo de proceso compuesto por ocho actividades (WfMC, 1999)

ha de realizar las actividades del proceso. La estructura organizativa es asimilada por el sistema de workflow, el cual asigna actividades a sus componentes, en lo que viene a denominarse "asignación de personal". Esta asignación puede modificarse en cualquier momento, ya que el sistema no asume ninguna estructura fija o permanente, lo que muestra que existe dinamismo en la gestión del proceso.

c) La infraestructura tecnológica (cuál de los recursos es utilizado): Una vez se conoce la actividad (qué) y la persona que la efectuará (quién), resta saber el recurso que se utilizará para ello (cuál). El punto de vista o dimensión de la infraestructura del proceso muestra la aplicación que servirá de soporte a la actividad y dónde se ejecutará (ejecución local o remota). La asignación de aplicaciones, al igual que los componentes de la estructura de la organización, puede modificarse según los casos y necesidades, pues tampoco se asume ningún tipo de estructura fija a este respecto.

La figura 3 muestra la unión de las dimensiones de un flujo de trabajo. Esta fusión se puede materializar mediante un cubo en el que sus lados visibles representan los componentes de la definición de un flujo, convergiendo todos ellos en un punto que supondría el entorno del sistema de workfow, en el que es posible la integración de la estructura organizativa, la infraestructura tecnológica, y la lógica del proceso o conjunto de actividades a desarrollar.

\subsection{Estructura de un sistema normalizado de flujo de trabajo}

Existe una gran heterogeneidad en el mercado en cuanto a productos workflow. Este hecho se debe principalmente, a que sus creadores se han guiado por sus propios criterios y objetivos a la hora de plantear sus

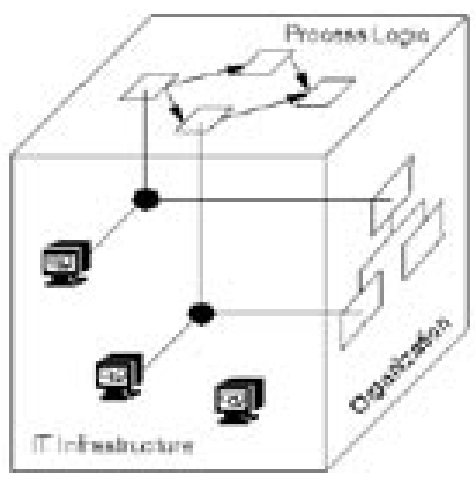

Fig. 3. El cubo del workflow (IBM, 199?)

Scire. $9: 2$ (jul.-dic. 2003) 37-59. 
funciones y características. Aún así, todos ellos coinciden en un mismo objetivo: la automatización dinámica de los procesos de la organización.

Con esta base, la WfMC ha establecido especificaciones para disponer de un modelo normalizado de sistema de workflow, con el fin de disponer de un estándar que termine con la heterogeneidad en este tipo de productos, y que permita a diferentes sistemas trabajar juntos en un mismo entorno, sin ocasionar problemas de compatibilidad. El resultado es el Modelo de Referencia de Workflow (figura 4), compuesto por una serie de interfaces y componentes genéricos que todo sistema workflow debe poseer. La estructura de este modelo está formada por una serie de componentes genéricos que aparecen en cualquier tipología de sistemas de workflow. La normalización tanto de estos interfaces, que representan los componentes genéricos, como los formatos de intercambio de datos entre ellos es lo que posibilita la interoperatibilidad entre productos heterogéneos.

A través de los interfaces y componentes principales que caracterizan al modelo de referencia (Hollingsworth, 1995), se vislumbra el funcionamiento en general de un sistema de flujo de trabajo. Son los siguientes:

a) Interfaz 1: Definición de procesos: La definición del proceso se refiere a la representación gráfica del mismo, especificando sus puntos de inicio y conclusión, las actividades que lo componen, los participantes a los que se asignan y los recursos tecnológicos necesarios para su realización (WfMC, 1999). Se trata del módulo a través del cual es posible definir gráficamente el flujograma del proceso, el cual contiene todos sus elementos. El objetivo de este interfaz es conseguir un modelo normalizado de definición de proceso, lo que posibilita que otros productos de workflow (ya sean aplicaciones de definición o ejecución de flujos), pueden trabajar con ella sin tener problemas de formato.

b) Interfaz 2: Aplicaciones cliente: Una aplicación cliente es un software que el participante o cliente del flujo utiliza para la interacción con el sistema de workflow, ejecutando las aplicaciones necesarias para llevar a término las actividades que tiene asignadas. La aplicación de las especificaciones correspondientes de este interfaz al sistema de workflow, permite que cualquier aplicación cliente - ya forme parte del mismo sistema, de otro diferente, o sea una aplicación independiente - se comunique eficazmente con éste para solicitarle aquéllos servicios que sean necesarios para procesar las actividades pertinentes.

c) Interfaz 3: Aplicaciones invocadas: Este interfaz contiene especificaciones referentes a APIs (Application Program Interface o Interfaz para el Programa de Aplicación) que permiten la comunicación eficaz entre el motor de workflow y aquéllas aplicaciones que éste ha de ejecutar para que sirvan de soporte a las actividades correspondientes. Son desarrolladas conjuntamente a las especificaciones del interfaz 2 , pero cada una tiene sus propios objetivos.

Scire. $9: 2$ (jul.-dic. 2003) 37-59. 


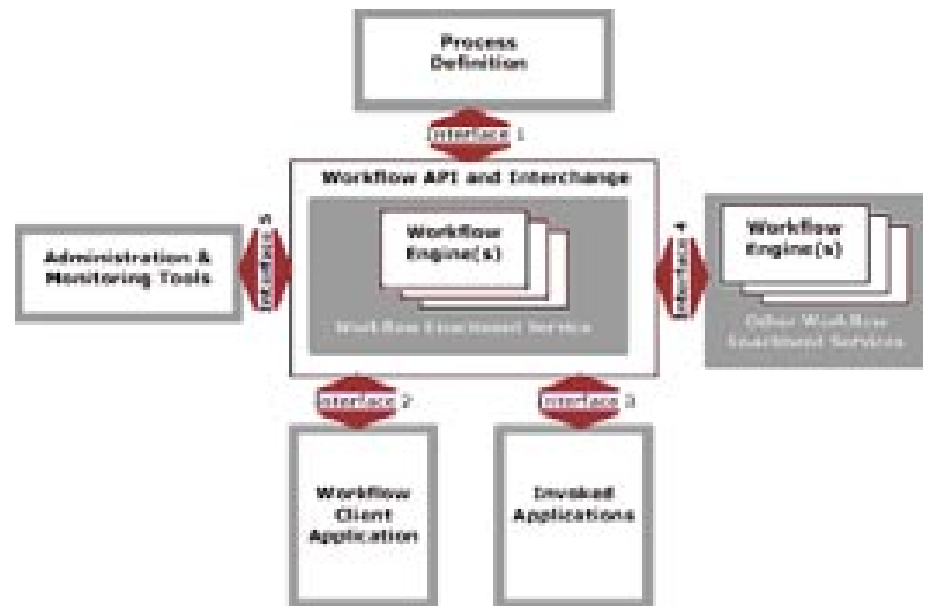

Fig. 4. Modelo de Referencia del Flujo de Trabajo (Hollingsworth, 1995)

d) Interfaz 4: Interoperatibilidad entre sistemas workflow: La interoperatibilidad tiene una gran relevancia para la Coalición, pues con esta función se consigue el intercambio de elementos de trabajo entre productos workflow diferentes. Las especificaciones de este interfaz tienen la misión de que los motores de workflow de dos o varios sistemas se comuniquen eficazmente para intercambiar definiciones de procesos, información sobre el estado de actividades, y se coordinen para ejecutar flujos, invocar actividades o subprocesos, etc.

e) Interfaz 5: Administración y monitorización de flujos: Contiene las especificaciones necesarias para que un sistema de workflow pueda trabajar en la gestión de flujos con cualquier tipo de aplicación de administración y monitorización. Con ello, se dispone de los medios necesarios para efectuar un continuo y constante seguimiento del proceso, conociendo el estado de cumplimiento de las actividades, si han sido reasignadas, la carga de tareas de cada usuario, etc., a la vez que se administran aspectos de ese proceso, como los permisos de acceso, las modificaciones en el flujograma, la reasignación de actividades, etc.

f) Motor del sistema de workflow: Es el componente del sistema que interpreta la definición del proceso (flujograma) y se ocupa de ejecutarla, a la vez que interactúa con aquellos recursos externos necesarios para procesar las actividades. Controla todo lo relacionado con la ejecución de las actividades del proceso, pues se ocupa de modificar sus posibles estados (iniciada, ejecutada, activa, suspendida, finalizada y completada). Se trata pues, de "un servicio software o motor, que proporciona el entorno de ejecución para cada instancia del flujo de trabajo" (Hollingsworth 1995).

Scire. $9: 2$ (jul.-dic. 2003) 37-59. 


\subsection{Beneficios de su aplicación en la automatización de bibliotecas}

Los sistemas de workflow permiten un alto grado de integración de los componentes de la organización que intervienen en el desarrollo de los procesos. Es precisamente esta integración la que permite que todos ellos trabajen coordinada y eficientemente en aras de los objetivos propuestos. A este respecto, Pyke (1998) afirma que "el workflow es el pegamento que une sistemas y entornos", y que "esta integración ha de cubrir todos los aspectos de los procesos de negocio". Desde un punto de vista genérico, la aplicación de sistemas workflow a los procesos reporta notables beneficios tanto por la mejora de los propios procesos (Pyke, 1998) como de la documentación implicada (Stark, 1998), entre los que destacan los siguientes:

a) Reducción del tiempo de respuesta: La ejecución de actividades es continua y constante, ya que se le asignan tiempos límite para su cumplimiento. Si se retrasa su cumplimiento, se envían notificaciones al usuario correspondiente, e incluso se reenvía a otro distinto si finalmente no se realiza.

b) Documentación de procesos: Este beneficio es implícito de los sistemas workflow, ya que para llevar a cabo la automatización del proceso, es necesario que se encuentre totalmente documentado, especificando todos sus elementos y datos correspondientes. Una vez el proceso es automatizado, la organización dispone de la documentación que lo describe.

c) Reducción de ciclos mediante la ejecución en paralelo: Permiten el establecimiento de flujos paralelos de actividades, de forma que su ejecución es más fluida. Así, una serie de actividades pueden realizarse simultánea. Luego, sus resultados confluirán en un mismo punto para continuar la ejecución del resto del proceso.

d) Reducción de la vulnerabilidad y los riesgos: El proceso se encuentra definido en su totalidad, aplicando una serie de reglas y excepciones a sus actividades, lo que elimina la posibilidad de fallos imprevistos durante su ejecución.

e) Reducción del uso del papel: Los documentos se gestionan de forma electrónica. Solo hay un ejemplar por documento, no existen copias de los mismos, aumenta la seguridad y se minimizan los riesgos y costes del transporte físico.

f) Documentos siempre disponibles: Se encuentran almacenados en el propio sistema, lo que agiliza su consulta y disponibilidad.

g) Siempre hay información disponible sobre el progreso de los procesos y sus actividades: Permite el seguimiento en tiempo real del estado de cumplimiento del proceso.

En el ámbito específico de la automatización de bibliotecas se pueden precisar también importantes ventajas específicas del workflow:

Scire. $9: 2$ (jul.-dic. 2003) 37-59. 
a) Permite pasar de un modelo de automatización de la colección, a la automatización de actividades y servicios de forma integrada (peticiones de búsqueda de información, programa de visitas, reuniones de trabajo, etc.)

b) Permite que la biblioteca defina en cualquier momento nuevos procesos, no contemplados en una fase inicial, en contraposición a los SIGB, que gestionan procesos cerrados. De esta forma, la biblioteca evita recurrir a programas externos para gestionar actividades no previstas, y por tanto, no se ve abocada a generar islas de automatización.

c) De la utilización de estos sistemas se genera el diagrama de flujo de los procesos. Al definir un proceso cualquiera, el sistema está generando toda la documentación de procedimiento asociada.

d) Al poner en práctica una determinada forma de proceder, se está contribuyendo a la ampliación de la normalización en los procesos bibliotecarios.

e) Al existir una total integración de los componentes y disponer de un módulo de administración y monitorización, permite una mejor gestión del personal, equilibrar las cargas de trabajo, detectar procesos saturados y supervisar el estado de realización de proyectos y trabajos en curso.

f) Posibilita la coordinación de diversos procesos, gracias a la función de interoperatibilidad, consiguiendo así un nivel de integración y cooperación global, tanto a nivel de una biblioteca como de un conjunto de ellas.

g) La normalización de los servicios garantiza unos mínimos de calidad de forma efectiva y eficiente.

h) El workflow puede tener una dimensión pública, mediante la posibilidad de conexión del usuario al flujo, permitiéndole conocer en todo momento la situación de sus demandas de servicios (búsquedas de información, reserva, reclamaciones, desideratas, etc).

\section{Diseño del flujo de trabajo para un proceso bibliotecario: desideratas}

Se presenta a continuación a modo de ejemplo una modelización de un proceso clásico en las bibliotecas, la desiderata - peticiones de ampliación de la colección, según las necesidades del usuario - tanto en forma gráfica (figura 5) como verbal. Se ha seleccionado este proceso porque es representativo tanto por los agentes implicados - usuarios, bibliotecas, librerías - como por la vinculación entre los numerosos procesos implicados - selección, adquisición, pre-catalogación, compras, préstamo, etc. La mecánica del proceso es la siguiente:

a) El proceso comienza al solicitar y cumplimentar el usuario el formulario de desiderata y entregarlo a la biblioteca, con lo que comienza el proceso en sí.

b) La biblioteca registra la desiderata en su base de datos correspondiente.

Scire. $9: 2$ (jul.-dic. 2003) 37-59. 


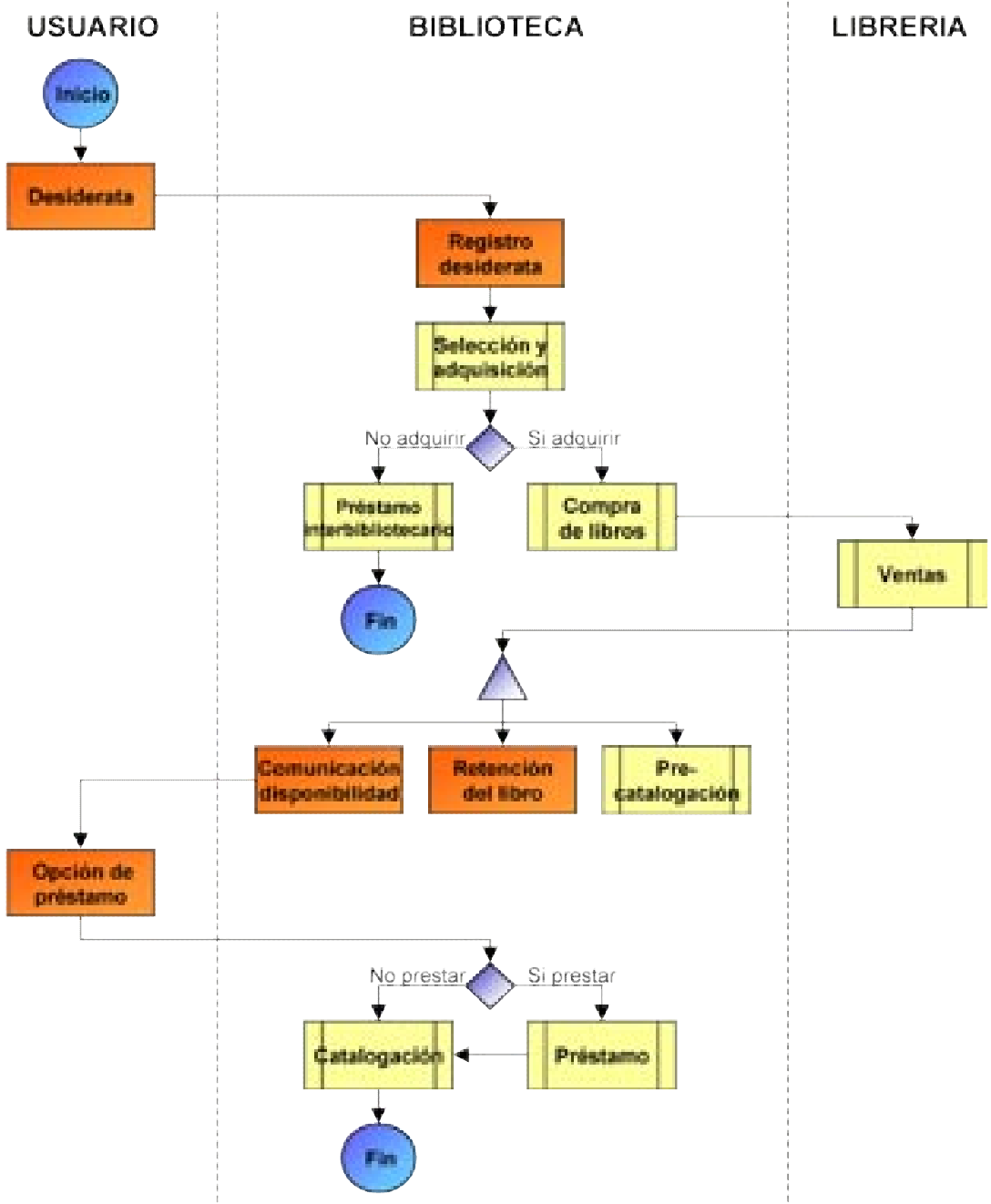

Fig. 5. Flujograma representativo del proceso bibliotecario "Desiderata"

c) Se inicia el subproceso de selección y adquisición, mediante el cual se decidirá la necesidad de adquirir el libro y la disponibilidad de prespuesto.

d) Si se decide no adquirir el libro solicitado, se activará el subproceso de préstamo interbibliotecario como alternativa, para no privar al usuario del acceso al libro. El proceso finaliza, puesto que el usuario ya dispone de él. 
e) Si la biblioteca está dispuesta a adquirirlo, se activa entonces el subproceso de compra, que dará lugar al inicio de otro subproceso, ventas, ya en la librería seleccionada.

f) El librollega a la biblioteca, y se ponen en marcha las actividades g), h) e i).

g) Se procede a la precatalogación del libro. Consiste en tomar sus datos identificativos e incluirlos en el OPAC, para acelerar su disponibilidad.

h) El libro se retiene, para que una vez que se ha pre-catalogado, no sea colocado en el estante correspondiente, sino que sea apartado en espera de que el usuario lo preste.

i) Se comunica al usuario la disponibilidad del libro que ha solicitado.

j) Una vez que el usuario sabe que el libro está en la biblioteca, puede tomarlo prestado o rechazarlo si ya dispone de él, ha perdido el interés, etc.

k) Si transcurrido un determinado tiempo, el usuario no solicita el libro, el sistema entiende que no le interesa y finaliza el proceso con la catalogación detallada.

1) Si el usuario decide acudir a la biblioteca y tomar en préstamo el libro, se iniciará el correspondiente subproceso de préstamo. Una vez el libro sea devuelto, se catalogará completamente y el proceso finalizará.

\section{Conclusiones}

- El sistema de información de la biblioteca no es una entidad cerrada en si misma, sino que por posee una capa exterior de servicio e información al usuario, que podemos denominar portal.

- El modelo conceptual de la automatización de bibliotecas las entiende como una organización compleja, productora de servicios al usuario y fundamentada en un sistema de información documental.

- En la biblioteca automatizada, la visión pública de los servicios y recursos de la biblioteca será el portal, y la visión interna será el sistema de flujo de trabajo para la producción del sistema de información, la organización de las tareas y la atención de los servicios.

- La conceptualización de los SIGB se debe realizar desde un doble enfoque: por un lado, como una herramienta para la realización de los procesos técnicos profesionales de la biblioteca, soportada por una aplicación de groupware especializada en gestión documental y por un sistema de gestión de flujo de trabajo; por el otro, como un producto de información y servicio al usuario, conceptualizado como un portal y una comunidad virtual. 
- El modelo de referencia de flujo de trabajo sirve como entorno de soporte para el sistema de información de la biblioteca por su carácter de software integrador.

- Las tecnología de flujo de trabajo puede funcionar junto con otras aplicaciones de groupware y recuperación de información, y otros formatos y protocolos normalizados de almacenamiento y recuperación de información, como el formato MARC y Z39.50.

- La definición detallada de todos los procesos que se desean automatizar con un sistema de flujo de trabajo proporciona procedimientos documentados exhaustivamente, reutilizables y normalizados.

- El sistema de flujo de trabajo permite un seguimiento eficiente, efectivo, constante e inmediato del estado de los procesos, lo que contribuye a la consecución de los planes de aseguramiento de la calidad.

- La formalización de los procedimientos, tanto de proceso técnico como de gestión de servicios al usuario, posibilitará la implantación de sistemas de gestión de calidad y la normalización.

\section{Notas}

(1) Beca financiada por la Fundación Séneca (Murcia), Centro de Coordinación de la Investigación, a través del programa de Formación del Personal Investigador.

(2) Sirva como reflexión final un apunte sobre la generación de valor añadido. Una vez ofertado el acceso a Internet gratuito para los usuarios, si buscamos un tipo de usuario que enriquezca a la biblioteca y que en ella pueda crecer personalmente, hay un laborioso camino que recorrer para que el gancho de Internet repercuta en el resto de servicios, atrayendo a los tecno-usuarios. Hay dos pasos que no pueden dejar de darse, y que es muy posible que encuentren bastantes obstáculos reales para producirse. Por un lado, ofrecer servicios de valor añadido a Internet - suscripciones, colección electrónica propia en red, asesoramiento cualificado-; y, por otro, espacios de valor añadido - acceso a prensa electrónica y bases de datos en la hemeroteca, navegador audiovisual en la mediateca, buzón de sugerencias informático, red electrónica en sala infantil, digitalización del fondo de consulta, terminales con tutoriales para la autoformación, etc.

(3) El acceso a los documentos electrónicos tangibles plantea problemas de manipulación, volumen y disponibilidad de puntos de consulta. Por ello, la tendencia es ofrecer el acceso a estos documentos a través de redes, mediante sistemas de volcado centralizado de información, basados en la tecnología Citrix Metaframe y Windows 2000.

\section{Bibliografía}

Barò, J.; Ontalba y Ruipérez, J. A. (2001) : Portales españoles: ¿demasiados productos para pocos clientes? // UOC. (Agosto 2001). <URL:<http://www.uoc.es/web/esp/art/ uoc/0107029/portales.html>. Consultado: 2001-09-20. 
Brophy, P. (2001). The library in the twenty-first century: new services for the information age. London: Library Association Publishing, 2001.

Crawford, W.; Gorman, M. (1995). Future libraries: dreams, madness, and reality. Chicago: American Library Association, 1995.

Garcia Gomez, J. C. (2001). Portales de Internet: concepto, tipología básica y desarrollo. // El profesional de la información. 10:7-8 (2001) 4-13.

González Lorca, J. (2001): Validez del estudio de la tecnología de flujo de trabajo para la organización automatizada del trabajo cooperativo. // Revista Española de Documentación Científica. 24:2 (abril-junio 2001) 198-211.

Hollingsworth, D. (1995). The Workflow Reference Model. Winchester: Workflow Management Coalition, 1995. URL: <http://www.aiim.org/wfmc/standards/docs/ tc003v11.pdf>. Consultado 1999-01-12.

IBM (1995). Workflow Resource Manager. URL: <http://as400bks.rochester.ibm.com/ cgi-bin/bookmgr/BOOKS/ID0H2000/id0h2000.boo>. Consultado: 1999-06-06.

Moreiro González, J. A. (1999) La distribución de los contenidos en la nueva sociedad informacional. // Caridad Sebastián, Mercedes (Coord.). La sociedad de la información: política, tecnología e industria de los contenidos. Madrid: Centro de Estudios Ramón Areces: Universidad de Carlos III, 1999. 312-316.

Oppenheim, C.; Smithson, D. (1999) What is the hybrid library? // Journal of Information Science. 25:2 (1999) 97-112.

Pyke, J. (1998). What is workflow enabling? // Workflow handbook 1997. Chichester: Wiley, 1998. 153-156.

Rusbridge, C. (1998). Towards de Hybrid Library. // D-Lib Magazine. (July/August 1998).

Saorín Pérez, T. (2001) : La biblioteca híbrida. // Tejuelo. 2 (2001).

Saorín Pérez, T.; García Gómez, J. C. (coords.). Los portales de Internet. Curso de promoción educativa. Facultad de Ciencias de la Documentación. Universidad de Murcia, Mayo 2001. URL: <http://www.um.es/gtiweb/portales>.

Saorín Pérez, T.; Sánchez Arce, M. V. (2001): Las comunidades virtuales y los portales como escenarios de gestión documental y difusión de información. // Anales de documentación. 4 (2001).

Stark, H. (1998) Understanding workflow. // Workflow handbook 1997. Chichester: Wiley, 1998, p. 5-25.

Workflow Automation Corporation. Workflow automation: new opportunities for dramatic IT results. Ontario (Canadá): Workflow Automation Corporation, c1998. URL: <http://www.workflow.ca/workflow.pdf>. Consultado: 2000-01-06.

Workflow Management Coalition (WfMC). Terminology and glossary. Winchester: WfMC, 1999.URL:<http://www.aiim.com/wfmc/standards/docs/glossy3.pdf>. Consultado:2000-06-07. 